\title{
Filigrane
}

Écoutes psychothérapiques

\section{Lire Freud et autres écrits. Entretien avec Jean-Michel Quinodoz}

\section{France Senécal}

Volume 21, numéro 2, automne 2012

URI : https://id.erudit.org/iderudit/1015201ar

DOI : https://doi.org/10.7202/1015201ar

Aller au sommaire du numéro

Éditeur(s)

Revue Santé mentale au Québec

ISSN

1192-1412 (imprimé)

1911-4656 (numérique)

Découvrir la revue

Citer ce document

Senécal, F. (2012). Lire Freud et autres écrits. Entretien avec Jean-Michel

Quinodoz. Filigrane, 21(2), 111-124. https://doi.org/10.7202/1015201ar d'utilisation que vous pouvez consulter en ligne.

https://apropos.erudit.org/fr/usagers/politique-dutilisation/ 


\section{Lire Freud et autres écrits. Entretien avec Jean-Michel Quinodoz}

\section{France Senécal}

nvité par la Société canadienne de psychanalyse à l'occasion du congrès annuel de 2012, le psychanalyste Jean-Michel Quinodoz (Société suisse de psychanalyse) a accepté d'accorder une entrevue à France Senécal pour la revue Filigrane. Le $D^{r}$ Quinodoz a souhaité nous entretenir de la thématique de son ouvrage Lire Freud (PUF, 2004) lors de cette entrevue réalisée le 31 mai 2012.

France Sénécal: Bonjour Jean-Michel Quinodoz. C’est un plaisir de vous accueillir. Pour commencer, puisqu'il y a peut-être des gens qui vous connaissent peu, voudriez-vous nous dire ce qui vous a amené à la psychanalyse? En effet, nous venons tous d'ailleurs... alors qu'est-ce qui fait que vous vous êtes engagé si profondément en psychanalyse?

Jean-Michel Quinodoz: Je dirais que ma vocation psychanalytique a été plutôt tardive parce que je ne connaissais pas du tout ce qu'était la psychanalyse durant mes études. Je voulais devenir médecin et je n'avais pas du tout l'intention de me lancer dans la psychanalyse. J'ai commencé en médecine interne, j'étais tout à fait passionné par la physiopathologie que l'on découvrait à l'époque. Mais je n'étais pas satisfait de traiter des patients somatiques car j'avais toujours l'impression que leur affection prenait son origine en amont de leurs symptômes. Je me suis ensuite orienté vers la psychiatrie et j'ai eu la chance de rencontrer à Genève le professeur Julian de Ajuriaguerra qui s'était entouré de psychanalystes. À leur contact, la psychanalyse a pris sens pour moi, aussi me suis-je orienté dans cette direction. J'ai cru d'abord qu'il s'agissait d'un hasard, mais après coup j'ai réalisé que je cherchais quelque chose de ce côté-là, sans vraiment le savoir. Depuis 1970 je travaille en pratique privée uniquement comme psychanalyste, avec des patients à raison de quatre séances hebdomadaires pour la plupart. À Genève en particulier, jusqu'au milieu des années 1990, nous avons vécu un âge d'or de la psychanalyse car, en plus des nombreux psychanalystes qui exerçaient en privé, la plupart des directeurs des services universitaires de psychiatrie étaient eux-mêmes psychanalystes. J'ai 
toujours eu le sentiment que nous devions être conscients qu'il s'agissait d'une situation privilégiée et que ce ne serait peut-être pas toujours ainsi.

FS: Malheureusement, vous aviez raison! Alors, si je comprends bien, vous avez utilisé un terme qui m'a beaucoup frappée parce que vous l'utilisez aussi dans vos écrits, c'est le terme de vocation, dont on connaît bien la connotation religieuse - et à la limite, négative, que cela peut avoir - mais cela parle aussi d'un engagement profond envers la psychanalyse. Et le parcours que vous me décrivez me semble relever d'une sorte de cheminement intérieur, peut-être aussi d'un manque qui effectivement ont fait qu'il y avait cette nécessité d'ouvrir quelque chose.

JMQ: Oui, tout à fait. Au fond, je me rends compte que j'ai toujours voulu transmettre à d'autres ce que j'avais découvert dans ma propre psychanalyse... transmettre une sorte d'étincelle lumineuse liée à la prise de conscience, à l'insight. J'ai tenté de la communiquer d'abord à mes patients, ensuite aux personnes désireuses de devenir psychanalystes et au-delà au grand public à travers mes conférences et mes écrits. Je pense important d'écrire la psychanalyse dans un langage clair et accessible pour les collègues autant que pour le grand public. Parce que Freud utilise un langage très proche du parler de tous les jours lorsqu'il écrit en langue allemande, sans néologisme et accessible à un large lectorat.

FS : Cela nous donne l'occasion de parler de ce beau livre que j'aime beaucoup et qui s'appelle Lire Freud. Vous me disiez qu'il est encore publié, traduit et qu'il circule encore beaucoup. À la lecture, on comprend pourquoi. Justement, on y retrouve ce parti-pris de clarté, de limpidité et, même si on a lu Freud, on apprend encore des choses. C'est passionnant parce que vous mettez des auteurs en écho à Freud. Pouvez-vous nous parler de ce qui a donné naissance à ce livre-là?

JMQ: En fait, au début de ma formation pendant les années 1970, on lisait très peu Freud dans notre société de psychanalyse. On connaissait des parties de son œuvre et pas d'autres, compte tenu en particulier de l'absence de ses œuvres complètes en langue française. Par contre, nous lisions des psychanalystes contemporains de l'époque, surtout français. Finalement, ce sont des candidats qui ont pris l'initiative de demander un séminaire de lecture de Freud et, personne ne s'étant annoncé, je me suis porté volontaire pour tenter cette aventure avec eux. Je ne voulais pas d'un séminaire classique dont je serais le leader, mais un séminaire où chacun participerait activement.

C'est ainsi que le séminaire s'est mis en place au cours duquel nous avons lu les principales œuvres de Freud à raison d'une rencontre chaque 15 jours 
sur un cycle d'une durée limitée à trois ans. Chaque participant s'engageait non seulement à lire les textes en suivant l'évolution chronologique de la pensée de Freud, mais également à rédiger une page situant le texte étudié dans son contexte historique et biographique, en repérant les concepts à mesure de leur apparition et en éclairant les textes freudiens à la lumière des psychanalystes postfreudiens. À la fin de chaque séminaire, l'un des participants en rédigeait le compte-rendu. Ce travail a demandé beaucoup d'engagement de la part des candidats, mais nous étions tous récompensés par la passion qui animait l'ensemble des participants du séminaire, y compris moi-même, travail qui s'est renouvelé chaque trois ans pendant une vingtaine d'années.

Pendant longtemps, je n'avais jamais pensé écrire à partir de mon expérience vécue dans ce séminaire, pensant surtout qu’il existe tellement d'ouvrages consacrés aux différents aspects de l'œuvre de Freud. Un jour cependant, j'eus l'idée que je pourrais offrir aux lecteurs une vue d'ensemble, chronologique de l'œuvre de Freud en faisant appel à des encadrés éclairant chaque œuvre sous des angles différents: biographique, historique, conceptuel et postfreudien. Cette idée m'est venue à partir d'un guide de voyage dont la variété des encadrés rendait compte des différents aspects de la vie du pays que je visitais, sous une présentation graphique attrayante et d'une lecture aisée. Selon les préférences, on peut lire l'ouvrage de manière linéaire, chapitre après chapitre en suivant l'ordre chronologique, ou en ordre dispersé, ce qui est une autre manière de parvenir à une vue d'ensemble.

La présentation graphique a fait beaucoup en faveur de l'attrait exercé par cette approche originale qui permet de rendre compte des 45 années au cours desquelles l'œuvre de Freud s'est peu à peu développée. Lire Freud est traduit actuellement en une quinzaine de langues, y compris l'hébreu, le japonais et le chinois. J'ai été moi-même surpris de l'intérêt qu'il a suscité pas seulement dans les milieux psychanalytiques, mais aussi dans les milieux universitaires, les lycées et auprès de beaucoup d'autres personnes intéressées par la psychanalyse. J'en suis ravi parce que l'écriture fait partie de ce à quoi je tiens beaucoup : continuer à faire connaître la psychanalyse qui, hélas, est encore souvent mise à mal à l'heure actuelle, bien qu'elle n'ait rien perdu de sa valeur.

FS: C'est pareil ici tout comme en France...

JMQ: Oui, comme un peu partout dans le monde.

FS: Dans ce livre, j'ai appris beaucoup de choses et je l'ai lu avec plaisir. Je ne m'attendais pas à ça du tout, je me suis dit: «bon je vais aller voir ce que ça donne» et ça a été une très belle surprise, ce Lire Freud. Alors, je vous en 
remercie. Mais votre engagement envers la psychanalyse se reflète également dans cet effort de traduction d'articles en français, là encore pour assurer la diffusion de la psychanalyse. Je crois que cela occupe une bonne part de votre temps maintenant.

JMQ: Favoriser la traduction de textes psychanalytiques a été et reste une activité que j'ai poursuivie tout au long de ma carrière et, après coup, je m'étonne du chemin parcouru. Après avoir été reçu comme membre de la Société suisse de psychanalyse en 1979, j'ai commencé par publier un Bulletin de la Société suisse de psychanalyse parce qu'il n'y en avait pas. Je pensais qu'il était très important de conserver des traces de ce qui se passe dans notre Société d'autant plus qu'elle est une société multilingue; la Suisse est en effet divisée en plusieurs régions où l'on parle français, allemand, italien et romanche. Dans le Bulletin, tous les articles sont traduits dans les deux langues principales, l'allemand et le français. Au début, les esprits chagrins nous ont annoncé que nous ne publierions qu'un seul numéro, le premier, et que les collègues s'en désintéresseraient ensuite. La raison? C'était, nous prédisait-on, parce ce que les Suisses écrivent aurait peu de valeur par comparaison avec nos collègues étrangers, notamment français, si brillants et si admirés. Maintenant, que le Bulletin de la Société suisse de psychanalyse est publié régulièrement depuis plus de 40 ans, deux fois par an, et il rend un énorme service de communication et de mémoire.

Cette expérience éditoriale initiale a certainement constitué un tremplin qui m'a conduit à devenir correspondant du Bulletin de la Fédération européenne de psychanalyse, de la Revue française de psychanalyse, enfin de ma désignation comme Editor for Europe de The International Journal of Psychoanalysis avec comme Editors-in-Chief David Tuckett, ensuite avec Glen Gabbard et Paul Williams de 1994 à 2003, enfin avec Dana Birksted-Breen et Bob Michels de 2009 à 2010. Dana est maintenant éditrice responsable de IJPA. The International Journal of Psychoanalysis a entre autres buts d'encourager les auteurs à se mettre à écrire, à soumettre leurs contributions à ce qu'on appelle la peer review, c'est-à-dire la « revue par les pairs» qui s'effectue de manière anonyme et permet un dialogue avec les auteurs tant sur le plan de l'écriture que sur celui des idées. J'ai eu vraiment beaucoup de plaisir à exercer cette responsabilité.

J'ai eu la chance de prolonger cette activité d'éditeur à partir de 2003, à la demande de Paul Williams et Glen Gabbard qui souhaitaient atteindre un lectorat psychanalytique qui n'a pas une connaissance suffisante de la langue anglaise pour lire aisément The International Journal of Psychoanalysis. Pour 
concrétiser ce projet, j'ai fait appel à de nombreux collègues européens provenant de différentes régions linguistiques et disposés à se lancer dans cette aventure, ce qu'ils ont fait et continuent à faire dans l'enthousiasme, ce dont je leur suis très reconnaissant. Il s'agissait de poursuivre en Europe une expérience qui avait débuté dans les années 1980 en Amérique latine où paraissaient les traductions en espagnol et en portugais d'une sélection annelle d'articles publiés précédemment en anglais dans The International Journal of Psychoanalysis. À partir de ce modèle, j'ai commencé par développer en Europe une version en français - L'Année psychanalytique internationale suivie successivement de versions en allemand, italien, russe, turc et depuis 2013 en grec (www.annualsofpsychoanalysis.com). La sélection des articles et leur traduction sont effectuées bénévolement par des collègues psychanalystes. Cette activité éditoriale me tient beaucoup à cour et, en ce qui concerne l'édition en français, je suis reconnaissant envers les membres du comité de rédaction qui proviennent de France, de Suisse, de Belgique et, tout particulièrement du Canada car Louis Brunet en est actuellement l'éditeur en chef, avec Marcel Hudon et André Renaud qui font partie du comité de rédaction.

FS: Cette collaboration avec The International m'amène à vous interroger sur le livre que vous avez publié sur Hanna Segal qui malheureusement est fort peu connue, du moins en milieu francophone. C'est là aussi un livre que j'ai beaucoup aimé parce que, bien sûr, c'est une série d'entrevues avec Hanna Segal, mais c'est très signé Jean-Michel Quinodoz, vous y êtes très présent. Non pas au point où Hanna Segal disparaît, mais il y a une sorte de complicité intellectuelle psychanalytique je dirais entre vous et elle parce qu'elle vous a beaucoup inspiré je crois.

JMQ: Cette complicité entre elle et moi vient de ce qu'Hanna Segal a conservé des liens étroits avec Genève où elle a passé quelques années durant son enfance avec ses parents. En 1933 en effet, son père avait dû quitter la Pologne pour des raisons politiques et il vint en Suisse où il travailla comme journaliste auprès la Société des Nations à Genève. C'est dans cette ville qu'Hanna Segal a appris la langue française et qu'elle a découvert la psychanalyse à travers la lecture des ouvrages psychanalytiques appartenant à la bibliothèque de son père. Par ailleurs, sa famille recevait parfois la visite de la psychanalyste polonaise Eugenia Sokolnicka installée à Paris qui avait contribué à fonder la Société psychanalytique de Paris. Parmi les ouvrages qui avaient le plus frappé la jeune Hanna, m’a-t-elle rapporté, figurent les travaux de Freud sur la pulsion de mort. En 1936, Segal retourna en Pologne 
pour entreprendre des études de médecine puis, en 1939, elle rendit visite à ses parents qui avaient alors quitté la Suisse pour s'installer à Paris; mais en raison de la déclaration de la guerre elle n'a pas pu retourner à Varsovie. En juin 1940, après quelques mois à Paris où Hanna poursuivit sa médecine, elle et ses parents trouvèrent refuge à Londres. En Angleterre Segal fréquenta les universités de Manchester puis d'Édimbourg où elle rencontra Fairbairn, puis elle retourna à Londres où elle entreprit une psychanalyse avec Melanie Klein et poursuivit la carrière psychanalytique fructueuse que l'on sait.

Ma rencontre avec Segal remonte aux années 1980 où elle commença à venir régulièrement à Genève afin de promouvoir le développement de la pensée kleinienne. C'est alors que j'ai suivi son enseignement sous la forme de séminaires et de supervisions individuelles et en groupe, à raison d'une fois par mois, enseignement qu'elle a poursuivi pendant une dizaine d'années. Paradoxalement, c'est elle qui m'a fait découvrir Freud en même temps qu'elle m'a introduit aux idées de Klein parce qu'elle possédait un talent particulier pour exposer la pensée de Freud, celle de Klein, la sienne propre ainsi que celles des autres psychanalystes d'une manière claire, sans être pour autant simpliste. Ce fut quelque chose de nouveau pour moi car, à l'époque, beaucoup d'écrits psychanalytiques me paraissaient hermétiques et d'une écriture parfois difficilement accessible. Pour moi, il a toujours été très important d'utiliser un langage courant pour parler de la psychanalyse, comme l'a fait Freud tout au long de son œuvre.

FS: Et en même temps, elle était aussi une clinicienne remarquable parce qu'elle a traité des gens très, très malades toute sa vie, ceux dont on disait que ce n'était pas des candidats à la psychanalyse. Elle ne s'en souciait pas. Là, je pense que Klein l'a beaucoup aidée, en fait, pas Klein, mais la pensée de Klein, à prendre à bras le corps, je dirais, ces patients-là qui n'étaient, le moins qu'on puisse dire, pas des patients classiques.

JMQ: Oui, tout à fait. J'ai pu apprécier la qualité de clinicienne de Segal à l'époque où elle était venue régulièrement à Genève de 1979 à 1989. En Suisse, l'école kleinienne était essentiellement représentée par Marcelle Spira, une psychanalyste suisse formée à Buenos Aires qui revint s'installer à Genève en 1955. Elle était relativement isolée c'est pourquoi Segal, qui parlait couramment français, est venue élargir les horizons de notre pratique en apportant l'éclairage des psychanalystes londoniens du Groupe Kleinien. Segal était très tolérante envers ses collègues - «non provocative» comme l'a qualifiée Melanie Klein dans l'une de ses lettres à Marcelle Spira - aussi cette capacité d'ouverture faisait qu'elle était volontiers acceptée par les collègues qui appar- 
tenaient à différents courants psychanalytiques. À cette époque, en Suisse romande, nous étions baignés dans un environnement psychanalytique fortement influencé par des collègues appartenant à la Société Psychanalytique de Paris, eux-mêmes influencés par Lacan sans pour autant le suivre, notamment sur le plan de sa technique des séances à durée variable.

Cela nous ramène à votre question concernant le traitement psychanalytique des patients dits «difficiles" par Segal. L'école française, en particulier les élèves de Lacan, mettaient en avant l'idée que la psychanalyse était principalement destinée au traitement de patients présentant les caractéristiques d'une névrose, c'est-à-dire des patients d'emblée capables d'accéder au symbolisme; cette conception était en partie fondée sur l'idée que le patient devait découvrir par lui-même le sens symbolique de son discours inconscient, ce qui expliquait qu'à l'époque beaucoup de psychanalystes avaient tendance à rester plutôt silencieux durant des périodes de plus ou moins longue durée. À ce propos, Marcelle Spira m’avait rapporté un jour que René Diatkine, psychanalyste parisien qui venait régulièrement à Genève, s'était vanté qu'il pouvait parfois rester une année en silence avec des patients... Selon les collègues appartenant à ce courant psychanalytique, les patients dont le fonctionnement psychique était dominé par des angoisses et des défenses primitives, préœedipiennes étaient considérés comme ne relevant pas d'un traitement psychanalytique de fréquence élevée, sur le divan, mais en face à face, une ou deux fois par semaine.

C'est dans ce contexte que Segal nous a permis de découvrir qu'il existait un monde inconscient au-delà de la névrose, accessible au traitement psychanalytique sans modification majeure du cadre, un monde qu'André Green de son côté avait commencé à ouvrir aux psychanalystes français à partir de sa propre conceptualisation. Ce qui m'avait particulièrement frappé dans la pratique clinique de Segal, c'est la manière dont elle nous apportait les outils techniques qui nous permettaient de comprendre et de favoriser le passage, l'articulation entre, d'une part, un univers mental primitif, caractérisé par un complexe d'Edipe avec des objets partiels et par un défaut de symbolisation - niveau de l'«équation symbolique », selon elle - et, d'autre part, un univers où prédomine la représentation symbolique et un complexe d'Édipe avec des objets totaux. Cette double approche qui articule deux niveaux du fonctionnement psychique en les intégrant est devenue pour moi une évidence que j'observe et met sans cesse en pratique dans mon travail quotidien.

FS: Chez tout un chacun. 
JMQ: Chez tout un chacun. Chez les patients névrosés aussi, pas seulement chez les patients au fonctionnement psychique dominé par des angoisses et des défenses primitives. Alors, pour moi ça a été une découverte importante et puis c'est comme ça que j'ai beaucoup apprécié la pensée de Segal et que... beaucoup plus tard je me suis dit que pour partager cette découverte avec d'autres, cela valait la peine d'écrire quelque chose à partir d'interviews que j'ai eues avec elle.

FS: Vous montrez toute la fécondité théorique et clinique du concept de la symbolisation. Justement, de ce que vous appelez «l'équation symbolique», enfin, ce qu'elle appelle «l'équation symbolique » et qui vous a permis de travailler autrement avec les rêves, et dont vous allez nous parler pendant ce congrès.

JMQ : Ce qui m'a paru vraiment important dans l'apport de Klein et de postkleiniens, comme Betty Joseph, Herbert Rosenfeld, Hanna Segal suivis actuellement par Britton, John Steiner ou Michael Feldman, ça a été de réaliser l'importance de la découverte d'une transition possible, d'un va-et-vient permanent entre position schizo-paranoïde et position dépressive, entre fonctionnement psychique primitif et fonctionnement évolué, entre symbolisme primitif et représentation symbolique. Comme vous le dites, chacun de nous peut observer à l'intérieur de lui ce double niveau de fonctionnement psychique. Freud a aussi montré en 1938, dans l'Abrégé de psychanalyse, qu'en chacun de nous coexistent un côté névrotique et un côté non névrotique du fonctionnement; les vues ultérieures de Klein sont un prolongement de ce clivage au sein de notre esprit dont Freud a eu l'intuition et qui a ensuite été développé de manière plus spécifique par Bion avec le concept de «partie psychotique et de partie non-psychotique de la personnalité».

FS: Quand vous reprenez toute la question du symbolisme chez Segal, dans votre travail sur le rêve, effectivement, vous avez une approche assez innovatrice du travail avec le rêve des patients.

JMQ: Effectivement, cette idée m'est venue progressivement au cours du travail avec mes patients. Je prenais parfois des notes en mettant un signe à côté du matériel clinique en me disant: notons ce rêve qui me surprend parce qu'il est beaucoup plus clair que la plupart des autres et qu'il semble apporter une solution à des problèmes que je m'étais régulièrement posés avec ce patient, mais dont je ne voyais pas la clé jusqu'à ce rêve. J'ai longtemps pensé que de nombreux collègues avaient dû faire les mêmes observations que moi et que ce type de rêve ne méritait pas une mention spéciale; peu à peu cependant, j'ai commencé à m'interroger : pourquoi ce type de rêve apporte-t-il 
une solution inattendue, insoupçonnée et hautement significative à une problématique du patient? J'avais aussi de ce type de rêve l'image d'un triptyque dont il manquerait l'un des panneaux, et qu'un jour on découvre soudain la partie qu'on croyait définitivement perdue, qui nous permet désormais d'avoir une vue d'ensemble. Par la suite, j'ai avancé des hypothèses pour expliquer la genèse de ce type de rêve et j'ai fait appel à des mécanismes primitifs sous-jacents, différents de l'interprétation classique du rêve, notamment sur la distinction entre défenses primitives et défenses évoluées, déni, clivage et refoulement, manque d'intégration et intégration psychique.

FS: Ce qui est intéressant de ces rêves aussi, c'est le moment où ils apparaissaient... c'est-à-dire que très souvent on se laisse fasciner par les contenus du rêve, mais vous soulignez, vous pointez même, quelque chose du moment où ces rêves apparaissent.

JMQ : Je les ai appelés « les rêves qui tournent une page » parce que ces rêves marquent un tournant dans le processus d'élaboration. Paradoxalement, ils peuvent être pris pour des rêves d'angoisse, et souvent c'est la première chose que nous dit le patient: "C'est épouvantable, j'ai fait un rêve horrible. J'en suis encore là, je ne terminerai jamais cette analyse!» De son côté le psychanalyste peut être également impressionné par le contenu angoissant du rêve, et cela m'est souvent arrivé. Avec l'expérience j'en suis venu à penser que, suivant le contexte, ce type de rêve peut être compris non pas comme étant l'expression d'une régression, mais plutôt comme le signe d'un progrès dans l'intégration psychique du rêveur. En cela, ils sont à distinguer des rêves d'évacuation du type cauchemar. Pour mieux comprendre la distinction que j'établis entre un rêve qui accompagne une phase d'élaboration - un "rêve qui tourne une page » - et un rêve qui signe un manque d'élaboration, je rapporte la discussion suivante qui a eu lieu entre des analystes-formateurs. Quelle attitude adopter lorsqu'un patient en fin d'analyse apporte un rêve dans lequel il tue son analyste? Est-ce un signe de régression montrant que le patient a besoin de poursuivre son analyse? Ou est-ce un signe de progrès qui montre que l'analyse peut se terminer? Les avis ont été très partagés et la conclusion a été qu'il s'agit avant tout de situer le rêve dans son contexte: le patient a-t-il évolué au point de parvenir à se représenter des aspects de son inconscient restés jusqu'alors non représentables, parce que déniés et clivés? Ou bien le patient a-t-il encore tendance à évacuer des aspects de lui-même qu'il n'est pas encore parvenu à élaborer? C'est la question qui se pose lorsqu'on se trouve en situation de décider si tel rêve appartient ou non à la catégorie d'un «rêve qui tourne une page». 
FS: Ce qui est intéressant aussi est que vous ne tirez pas nécessairement ce genre de rêves du côté soit de la décharge ou soit du côté d'une régression, parce que c'est vécu souvent par les patients comme une régression, donc, quelque chose de très angoissant.

JMQ: La première réaction du patient - et souvent aussi le nôtre, comme analyste - peut être de se dire: «Ce rêve montre que je fais marche en arrière! » En fait, lorsqu'on prend en compte le contexte onirique dans son ensemble, on peut constater qu'un «rêve qui tourne une page» offre une représentation visuelle des fantasmes organisateurs inconscients sous-jacents à la problématique du rêveur, à la manière dont se rassemblent les pièces d'un puzzle pour former une image globale.

FS: Et qui témoignerait, diriez-vous, d'un passage qui n'était pas très clair, mais qui tout à coup met en image qu'il y a quelque chose de la position dépressive qui s'élabore.

JMQ: Tout à fait. C'est la position dépressive qui permet que se ramassent, se rassemblent dans une synthèse les morceaux du puzzle jusque là fragmentés, soumis à des dénis, à des clivages, à l'identification projective, et qui soudain forment un ensemble cohérent. Le progrès que permet l'élaboration de la position dépressive conditionne la capacité de se représenter une telle synthèse d'une manière symbolique.

FS : D'ailleurs, vous liez beaucoup la créativité à l'élaboration de la position dépressive.

JMQ: Oui, tout à fait.

FS: Hanna Segal a beaucoup travaillé cette question, que vous reprenez non pas tellement du côté de l'art, mais du côté justement du rêve. Comme quelque chose qui soutient la créativité parce qu'on peut dire de ces rêves-là que ce sont des rêves créateurs.

JMQ: À ce propos, on a souvent tendance à parler des symptômes en termes négatifs, pas seulement chez les patients souffrant d'une névrose mais aussi chez les patients borderline, psychotiques ou psychosomatiques. Cependant, nous pouvons regarder les symptômes sous un angle différent, sous l'angle de la création par le patient de défenses dans le but d'éviter l'angoisse. De ce point de vue, on ne peut qu'admirer la façon dont certains patients sont capables de créer des symptômes extrêmement complexes, prenant des chemins malicieux et détournés de sorte qu'on a beaucoup de peine à les reconnaitre et à les décrypter. Je crois que cette créativité fait aussi partie de la maladie car la pathologie possède une dimension créatrice qui n’est pas seulement négative, et il s'agit de prendre en compte sa richesse et sa diversité pour pouvoir l'interpréter. 
FS: D'ailleurs, je ne sais plus si c'est vous ou Hanna Segal qui, parlant de la création artistique et de la sublimation, dit d'un patient qu'elle avait: lui, sa sublimation, ça avait été de devenir fou... Bon, c'est un peu étonnant. Ça met la sublimation à mal un peu, mais ça décoiffe un peu aussi.

JMQ: Je pense que c'était aussi l'idée de Freud quand il parle du délire comme d'une création du patient. Cette «néo-réalité» constitue une création à travers laquelle on parvient à découvrir et analyser les secrets de la construction inconsciente du délire.

FS: Alors, puisque qu'on parle de créativité, on pourrait aller d'un côté un petit peu plus ludique de ce livre sur Hanna Segal. Il me semble en effet très personnel, c'est-à-dire que ce n'est pas un livre traditionnel «sur», mais que vous y êtes très présent. Par exemple, vous y évoquez une expérience que vous aviez eue en visitant une exposition de Magritte.

JMQ: Ah oui! J'avais lu les travaux de Segal sur l'impulsion créatrice et l'émotion esthétique mais j'ai mis du temps à découvrir leur importance et leur signification. J'ai mieux compris ses hypothèses il y a quelques années, à l'occasion d'une rétrospective Magritte au Centre Pompidou à Paris. J'aime les expositions qui présentent une œuvre selon un ordre chronologique parce qu'on peut y déceler le fil rouge qui parcourt la vie de l'artiste... Ainsi, c'est en lisant les notices biographiques de Magritte que j'ai réalisé qu'à l'âge de 14 ans, il avait perdu sa mère qui s'était suicidée en se jetant à l'eau lors d'une crise schizophrénique... En revisitant l'exposition depuis le début avec cet épisode dramatique à l'esprit, j'ai pris conscience que la plupart des toiles exposées portaient des traces du deuil lié à la perte de la mère de l'artiste. Par exemple, l'élément eau est presque partout présent. La plupart des toiles évoquent une atmosphère d'un drame mystérieux, ou portent la marque de la mort sous forme de la pierre ou du marbre qui forment la matière de ces bustes ou de ces fragments de corps féminins morcelés, etc. L'absence et la mort sont évoquées par la silhouette vide d'un personnage disparu, découpant un trou noir dans une porte. On y voit représenté l'étouffement à travers cette pomme envahissant toute une chambre. L'œuvre de Magritte a alors pris un sens nouveau à mes yeux...

Magritte était hostile envers la psychanalyse car il prétendait que l'analyse n'avait absolument rien à dire sur sa production artistique qui, selon lui, était une tentative de représenter « le mystère de la vie»... Il avait même tenté une psychanalyse, mais il n'avait pas poursuivi et il était resté critique visà-vis cette approche comme s'il s'en défendait. Redoutait-il peut-être de découvrir quelque chose d'angoissant en lui-même? Il souffrait d'obsessions, 
de rituels et d'angoisses. Par contre, il a connu des moments lumineux qui se sont traduits dans ces toiles sereines qui représentent une maison noire comme si elle était dans l'obscurité de la nuit, contrastant avec un ciel de plein jour. Ici encore c'est le contraste entre la nuit et le jour... et l'eau y est aussi présente dans le réverbère qui se reflète dans une flaque d'eau.

Alors, est-ce que Magritte a quelque chose à voir avec Segal ? Segal écrit son article sur l'origine de l'impulsion créatrice à l'âge de 30 ans, en partant des idées de Klein sur le deuil, la dépression et la position dépressive. Pour elle, l'impulsion créatrice naît de la sensation à l'intérieur de soi d'un chaos menaçant, d'un sentiment angoissant que notre propre monde est en train d'être détruit, réduit en pièces. De cette menace interne naît un besoin de mettre de l'ordre dans ce chaos, de le dépasser et de lui donner un sens: pour Segal, l'art naîtrait de ce chaos primitif et de la recherche d'une issue. Que ce soit en peinture, avec Magritte, ou que ce soit en littérature, avec Proust et $\grave{A}$ la recherche du temps perdu, ou dans n'importe quelle forme d'art, il s'agirait d'une tentative de surmonter, d'élaborer la position dépressive. Cette impulsion créatrice porterait toujours la marque personnelle de l'artiste et de la manière dont il cherche à résoudre son chaos intérieur, à la communiquer au public à travers sa forme d'art.

Bien entendu, ce que décrit Segal n'est pas applicable à toutes les formes d'art et, par exemple, ses idées ne s'appliquent que partiellement à l'art contemporain qui est très différent de l'art classique. Cependant, je pense que les idées de Segal sur la créativité restent l'un des mobiles inconscients majeurs de l'impulsion créatrice et l'œuvre de Magritte constitue pour moi un magnifique exemple des hypothèses de Segal que je retrouve chez de nombreux créateurs.

FS : Cela donne aussi un éclairage intéressant sur l'émotion esthétique qu'on peut éprouver parce que, restons dans le domaine de la peinture, effectivement c'est différent pour chacun, mais devant certaines toiles, on dirait que les artistes ont le don de nous mettre en contact avec notre propre chaos intérieur, et ce dans une sorte de mouvement de réparation. C'est ce qui fait que c'est si touchant parce qu'on ne reste pas seulement ébranlé et dans cette zone chaotique qui est si effrayante, on est témoin de ce qu'on peut en faire. Ce qui n'est pas donné à tout le monde en tout cas.

JMQ: Oui, c'est quelque chose qui touche chacun de nous; personnellement j'ai vraiment compris les idées de Segal à travers une expérience personnelle. Je viens d'une famille originaire d'un petit village de montagne, La Sage, situé en Valais à 1700 mètres d'altitude. J'avais une tante, une paysanne de mon- 
tagne qu'on avait surnommée Marie des Collines à cause du Café des Collines qu'elle avait fondé, un établissement à la fois café littéraire et café philosophique avant la lettre. Elle, que j'ai toujours vue vêtue du costume traditionnel, y recevait régulièrement des écrivains, des peintres et des musiciens en séjour à La Sage, comme par exemple le compositeur Henri Dutilleux et son épouse, la pianiste Geneviève Joy, de grands amis de ma tante. Après son décès, à la fin des années 1970, je me disais parfois: «De quelle manière pourrait-on laisser une trace en souvenir de ma tante et de son monde de l'époque?»

J'ai pensé demander d'abord à des artistes ou à des écrivains qui l'avaient connue de porter témoignage de sa personnalité à travers leur art, mais j'y ai finalement renoncé. Un jour, le déclic s'est produit lorsque j'ai lu dans un journal un portrait tellement déformé de ma tante par un journaliste pourtant bien intentionné, que j'ai ressenti que le monde de ma tante et celui de cette époque était en train de sombrer dans l'oubli et allait disparaître. Je me suis alors dit que puisque mon talent c'est écrire, pourquoi ne pas prendre ma plume et raconter l'histoire de cette tante exceptionnelle. C'est ainsi que j'ai senti monter en moi l'impulsion qui m'a permis de rédiger avec beaucoup d'émotion un ouvrage que j'ai intitulé Marie des Collines qui continue à être très apprécié, surtout de ceux qui fréquentent nos villages traditionnels de haute montagne. A posteriori, j'ai pris conscience que j'avais été à la fois saisi du vertige de voir une partie de mon monde intérieur menacé de destruction et du désir de le restaurer qui s'en est suivi, suivant en cela l'hypothèse de Segal.

Pour conclure, j'aimerais vous faire part de la surprise que j'ai eue en 2005, en me promenant à New York, lorsque j'ai découvert une citation de Segal affichée sur un panneau faisant face à Ground zero! Cette phrase, qui évoquait la menace de destruction intérieure et l'impulsion réparatrice, avait été choisie par une art-therapist pour être affichée à côté des autoportraits de nombreux enfants des écoles new yorkaises qui exprimaient ainsi l'émotion que leur avait fait ressentir la destruction des tours jumelles...

FS: Nous pourrions peut-être terminer l'entrevue en lisant cette citation. Je vais la lire en français. «C'est quand le monde à l'intérieur de nous est détruit, quand il est dans un état de mort et sans amour, quand les êtres aimés de nous sont en morceaux et que nous sommes nous-mêmes réduits au désespoir, c'est alors qu'il nous faut recréer notre monde de toutes pièces, rassembler les morceaux, insuffler la vie dans les fragments morts, recréer la vie.»C'est absolument magnifique. Je vous remercie de m'avoir permis, à la 
fois de vous connaître mieux, mais aussi de pénétrer un peu dans l'univers de Hanna Segal que je trouve extrêmement riche.

JMQ: Merci à vous aussi.

\section{Principales publications de Jean-Michel Quinodoz Articles}

2012, The European annuals of the International Journal of Psychoanalysis. The benefits of linguistic diversity, Psychoanalytic thinking, International Journal of Psychoanalysis, 93, 219238.

2010, How translations of Freud's writings have influenced French, Psychoanalytic thinking, International Journal of Psychoanalysis, 91, 695-716.

2009, Melanie Klein's letters adressed to Marcelle Spira (1955-1960), International Journal of Psychoanalysis, 90, 1340-1365.

2008, Construire et/ou interpréter? Discussion du rapport de Michèle Bertrand, Revue française de psychanalyse, 72, 155-163.

2006, Thinking Under Fire, International Journal of Psychoanalysis, 87, 1-10

Livres

2008, À l'écoute d'Hanna Segal. Sa contribution à la psychanalyse, Paris, PUF.

2005, Marie des Collines, Genève, Slatkine.

2004, Lire Freud. Découverte chronologique de l'œuvre de Freud, Paris, PUF.

2001, Les rêves qui tournent une page. Rêves d'intégration à contenu paradoxal régressif, préface d'Hanna Segal, Paris, PUF.

1991, La solitude apprivoisée. L'angoisse de séparation en psychanalyse, préface d'Hanna Segal, Paris, PUF ( $4^{\mathrm{e}}$ édition 2010). 